\title{
Amelioration Du Rendement Du Mil Par L'association Avec Le Niebe En Zone Sahelienne
}

\section{Toudou DaoudaAbdoul Karim}

Faculté des Sciences et Techniques, Université Abdou Moumouni, Niamey, Niger

Atta Sanoussi

Centre Régional AGRHYMET, Niamey, Niger

Hamidou Falalou

International Crop Research Institute for the Semi-Arid Tropic

(ICRISAT),Niamey, Niger

Inoussa Maman Maârouhi

Bakasso Yacoubou

Faculté des Sciences et Techniques, Université Abdou Moumouni, Niamey, Niger

\section{Saadou Mahamane}

Faculté des Sciences et Techniques, Université de Maradi,Maradi (Niger).

\begin{abstract}
In Niger, agricultural production is based mainly on the association and rotation grains / legumes with generally low yields. To improve these yields, seeding effects and cowpea genotypes in combination with millet were studied in 2014 and 2015 in the Sahel region of Niger. The experimental device is a split plot design with three replications. cowpea genotypes were large plots, and two seeding (1: 1 and 1: 2, millet and cowpea) in small plots. In addition, four parcels of pure millet grown in the past two years have been used as control. Analysis of variance revealed that for cowpea, biomass yield, seed, weight of hundred seeds and seed harvest index were significantly affected by the genotypes for two years. However, there is no significant effect on the density and cowpea genotypes on grain yields of millet. In 2014, the biomass yield millet density 2 was lower than the density of one. The density 2 increased yields of cowpea and millet slightly diminish those.
\end{abstract}

Keywords: association, rotation, yield, plant density, Niger 


\section{Résumé}

$\mathrm{Au}$ Niger, la production agricole est essentiellement basée sur l'association et la rotation céréales/légumineuses avec des rendements généralement faibles. Afin d'améliorer ces rendements, les effets de densités de semis et de génotypes de niébé en association avec le mil ont été étudiés en 2014 et 2015 en zone sahélienne du Niger. Le dispositif expérimental est un split plot à trois répétitions. Les génotypes de niébé étaient en grandes parcelles, et les deux densités de semis ( $1: 1$ et $1: 2$, mil et niébé) en petites parcelles. Par ailleurs, quatre parcelles de mil pur cultivées au cours des deux années ont été utilisées comme témoin. L'analyse de variance révèle que pour le niébé, le rendement en biomasse, en graines, le poids de cent graines et l'indice de récolte en graines ont été significativement affectés par les génotypes pendant les deux années. Cependant, il n'y'avait aucun effet significatif de la densité et de génotypes de niébé sur les rendements en grains du mil. En 2014, le rendement en biomasse de mil à la densité 2 était inférieur à celui de la densité 1 . La densité 2 augmentait les rendements du niébé et diminuent faiblement ceux du mil.

Mots clés: association, rendement, densité de semis, Niger

\section{Introduction}

Au Niger, le mil (Pennisetum glaucum L.) qui constitue la base de l'alimentation est la principale céréale cultivée avec des rendements très faibles (moins de $500 \mathrm{~kg} / \mathrm{ha}$ ) en raison de la pauvreté des sols et du faible taux d'utilisation de la fertilisation organique et minérale (JAICAF, 2009).Le niébé (Vigna unguiculata (L) Walp) et l'arachide (Arachis hypogaea L) sont les principales légumineuses cultivées en association ou en rotation avec le mil. En effet, les pratiques traditionnelles de la gestion de la fertilité des sols impliquent le recyclage des matériaux organiques comme les résidus de culture ou le fumier et en utilisant la rotation ou l'association avec les légumineuses (Bationo et al., 1998 ; Yamoah et al., 2003).

Le niébé et 1'arachide présentent un grand intérêt agronomique nutritionnel et économique pour les petits producteurs. Cependant, leur niveau de production est faible et considérablement affecté par les variations climatiques et la pauvreté en éléments nutritifs des sols.

Les légumineuses sont reconnues pour leur capacité à améliorer la fertilité du sol et notamment son statu azoté (Bado, 2002) à lutter contre les mauvaises herbes (Sarunaite et al., 2012). Elles peuvent également favoriser l'acquisition du phosphore par les céréales par des processus rhizosphériques (Elodie, 2012). Par ailleurs, les légumineuses offrent la possibilité de diversifier les systèmes fourragers, de sécuriser la production agricole et du 
bétail en garantissant la qualité des fourrages et la réduction des intrants (Naudin et al., 2009).

Pour une optimisation des modalités culturales de céréaleslégumineuses, le choix variétal et la densité de semis restent de ce fait une étape cruciale (Jérôme et al., 2013) bien qu'ilspeuvent dépendre d'un certain nombre de facteurs comme les traditions locales (Singh et Ajeigbe, 2000). En effet, les variétés que proposent les chercheurs dont la priorité est l'augmentation des rendements ne corroborent pas forcement le choix des paysans qui cherchent à réduire la fréquence d'échec de la culture, c'est-àdire obtenir des rendements supérieurs à un rendement minimum (Caroline, 2006). Mais en général, les densités de légumineuses utilisées en association avec le mil sont faibles et leur contribution à la fixation de l'azote pourrait être négligeable par rapport à une rotation où la culture suivante pourrait bénéficier de l'effet résiduel de l'azote fixé par la légumineuse (Bationo et Ntare, 2000). Or des possibilités existent pour améliorer la productivité des légumineuses et céréales en utilisant des génotypes adaptés et des techniques culturales appropriés et applicables en milieu paysan (Atta et al., 2011).

L'objectif principal de cette étude est d'identifier les génotypes de niébé permettant d'optimiser les rendements, dans l'association mil/ niébé.

\section{Materiel et methodes}

\section{Site de l'étude}

L'expérimentation a été conduite en milieu paysan au cours des saisons des pluies 2014 et 2015 à Boki (Say) situé à $75 \mathrm{~km}$ de Niamey. Le climat est de type sahélien. Les sols sont sableux, profonds, et naturellement pauvres en éléments nutritifs. Le précédent cultural est une jachère de 4 années.

La moyenne des précipitations de 1983 à 2015 était de $540 \mathrm{~mm}$. Le cumul de précipitations était de $577 \mathrm{~mm}$ en 2014 et $539 \mathrm{~mm}$ en 2015 . La figure 1 donne la distribution décadaire des précipitations pour les deux années.

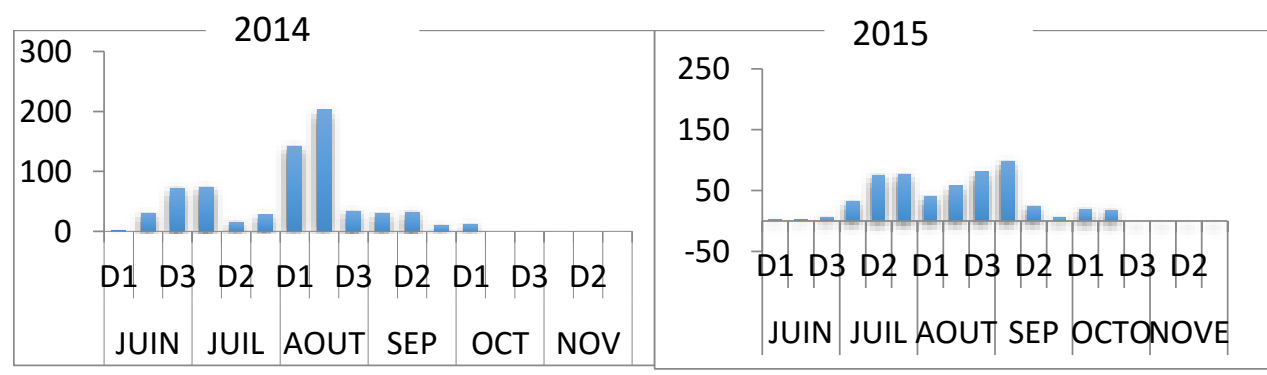

Fig 1. Distribution décadaire des précipitations (en mm) en 2014 et 2015 à Boki (Say). 


\section{Matériel végétal}

La variété de mil ICMV IS 99001 qui a un cycle de 90 jours a été mise en association avec dix génotypes de niébé .Le tableau 1 donne l'origine et la précocité des génotypes de niébé utilisés.

Tableau 1 : Origine et précocité des génotypes de niébé

\begin{tabular}{cccc}
\hline Nom & Origine & Précocité & Réponse à la sécheresse \\
\hline ISV128 & ISC Niger $^{1}$ & - & - \\
IT93K-503-1 & IITA Nigeria & Moyen & Tolérant \\
IT96D610 & IITA Nigeria & Moyen & Tolérant \\
Mouride & ISRA Sénégal ${ }^{2}$ & Précoce & Tolérant \\
& & & \\
IT90K-284-2 & IITA Nigeria ${ }^{3}$ & Moyen & Sensible \\
Tiligré & - & - & - \\
ISV20 & ISC Niger & - & - \\
IT98K-428-3 & IITA Nigeria & Moyen & Tolérant \\
IT18E18 & IITA Nigeria & Précoce & Sensible \\
Suvita2 & INERABurkina & Moyen & Tolérant \\
\hline \multicolumn{5}{c}{${ }^{1}$ ISC: ICRISAT SahelianCentre } \\
\multicolumn{5}{c}{ ISRA: Institut Sénégalais de Recherches Agricoles } \\
& ${ }^{3}$ IITA:International Institute for Tropical Agriculture \\
\hline
\end{tabular}

\section{Dispositif expérimental}

Le dispositif expérimental est un split plot avec trois répétitions. Les génotypes de niébé étaient en grandes parcelles, et les densités de semis en petites parcelles. Chaque parcelle principale, de dimension $17,5 \mathrm{~m} \times 11,5 \mathrm{~m}$ $\left(201,25 \mathrm{~m}^{2}\right)$ était subdivisée en 20 parcelles élémentaires (10 génotypes $\mathrm{x}$ deux densités). La parcelle élémentaire a une superficie de $6 \mathrm{~m}^{2}(4 \mathrm{~m} \times 1,5$ $\mathrm{m})$.

Les traitements (génotypes et densités de semis) ont été disposés au hasard dans chaque répétition. Les parcelles élémentaires successives sont espacées de $50 \mathrm{~cm}$ et les répétitions d'un mètre.

Le mil a été semé le 16 août en 2014, et le 23 juillet en 2015 avec un espacement entre les poquets de $80 \mathrm{~cm}$ et un écartement entre les lignes de 75 $\mathrm{cm}$.

Les 10 génotypes de niébé ont été semés à deux densités.

- D1: une ligne de niébé intercalée avec une ligne de mil. L'espacement entre les poquets de niébé était de $30 \mathrm{~cm}$ avec un écartement entre les lignes de $50 \mathrm{~cm}$. Chaque parcelle élémentaire contient 3 lignes de mil et 2 lignes de niébé.

D2 : 2 lignes de niébé pour une ligne de mil : Les mêmes écartements en D1 ont été gardés pour le niébé. Chaque parcelle élémentaire contient 3 lignes de mil et 4 lignes de niébé. 
Le niébé a été semé le même jour que le mil à raison de deux graines par poquet, démarié par la suite à un plant par poquet.

Les mêmes positions des variétés de niébé ont été gardées dans les parcelles en 2014 et en 2015.

\section{Données mesurées}

La récolte a été effectué sur un carrée de rendement de 2,4 $\mathrm{m}^{2}(1,6 \mathrm{~m}$ $\times 1,5 \mathrm{~m}$ ) formé de 14 plants de niébé pour la densité 1 , et 28 plants pour la densité 2.Pour les deux densités 9 plants de mil ont été récoltés. Les fanes du niébé ont été coupées au ras du sol.

Après séparation de la biomasse (tiges + feuilles) et gousses du niébé, puis séchage complet à l'ombre, les rendement en biomasse sèche et en graines ainsi que le poids de cent graines ont été déterminés.

L'indice de récolte (IR) des grains du niébé a été calculé par la formule de Manfred Huehn (1993) suivante :

IR $(\%)=$ Matière sèche des graines $\times 100 /$ (matière sèche totale)

La biomasse du mil (tiges + feuilles) et les épis ont été séparées. Après séchage, les rendements en biomasse sèche et en grains après battage ont également été déterminés.

\section{Analyse des données}

L'analyse de variance a été faite à l'aide du logiciel JMP9 version 9.0. Après vérification de la normalité, les données ne suivant pas une distribution normales ont subi une transformation logarithmique. La séparation des moyennes pour les différents paramètres mesurés a été réalisée par le test de Student Newman Keuls au seuil de $\alpha=5 \%$.

\section{Resultats et discussion \\ Résultats \\ Rendements du mil}

Les résultats des rendements grains et biomasse sèche du mil sont présentés dans le tableau 2. Il n'ya aucun effet significatif du génotype de niébé d'une part et de la densité d'autre part sur les rendements grains et biomasse sèche du mil en 2015.En 2014, on note cependant un effet significatif du génotype de niébé sur les rendements grains et un effet densité sur la biomasse du mil. Il ya une forte interaction génotype $\times$ densité sur le rendement en grains et en biomasse du mil en 2015. L'association du mil avec le niébé a diminué le rendement grains du mil en 2014.

En 2015, le mil en culture pure a produit plus de matière sèche avec plus de grains en association avec certains génotypes (ISV20, IT82E18, IT90K-284-2, IT96D-610, Suvita2, et Tiligré) et moins pour d'autres (ISV128, IT93K-503-1, IT98K-428-3 et Mouride). Pour la densité de semis, 
les rendements en grains et biomasse sèche du mil étaient légèrement plus élevés en densité 1 qu'en densité 2 . L'augmentation de la densité de niébé en association, a donc réduit le rendement en biomasse sèche du mil.

\section{Rendement du niébé}

Le rendement moyen en biomasse de 10 génotypes a été de 721,8 $\mathrm{kg} / \mathrm{ha}$ en 2014 et 829,91 en 2015 . Le rendement en biomasse a été significativement affecté par les génotypes d'une part et la densité de semis d'autre part pendant les deux années (Tableau 3). L'augmentation de la densité de semis a augmenté le rendement moyen du niébé en biomasse pour les deux années avec des valeurs supérieures en 2015.

Au niveau des génotypes, en dehors des génotypes qui ont donné des rendements médiocres en 2015, respectivement et,

En 2015, le rendement en biomasse de tous les génotypes étudiés était sensiblement égal ou supérieurs à $500 \mathrm{~kg} / \mathrm{ha}$, à l'exception de IT98K428-3 (443,96 kg/ha)et Mouride (376,46 kg/ha). Le rendement moyen maximal a été enregistré pour les génotypes Tiligré $(1076,74 \mathrm{~kg} / \mathrm{ha})$, ISV128 $(1047,19 \mathrm{~kg} / \mathrm{ha})$, en 2014 et IT93K-503-1 (1374,27 kg/ha), ISV20 (1226,08 $\mathrm{kg} / \mathrm{ha})$, ISV128 (1211,08 kg/ha), et Tiligré $(1089,38 \mathrm{~kg} / \mathrm{ha})$ en 2015.

Le rendement moyen en graines des génotypes a été de $41,62 \mathrm{~kg} / \mathrm{ha}$ en 2014 et $62,28 \mathrm{~kg} / \mathrm{ha}$ en 2015 avec une différence très significative entre les génotypes en $2014(p<0,01)$ et en $2015(p<0,0001)$ (Tableau 3$)$.

\section{Effet de la densité et de l'association sur les rendements graines, l'indice de récolte et le poids de $\mathbf{1 0 0}$ graines du niébé.}

Le rendement moyen maximal de niébé a été obtenu avec la densité 2 pour les deux années d'essai. Au niveau génotype, le rendement maximal en 2014 a été obtenu par les génotypes Suvita2 $(92,69 \mathrm{~kg} / \mathrm{ha}), \operatorname{ISV} 128(83,68$ $\mathrm{kg} / \mathrm{ha}$ ) et Tiligré $(66,85 \mathrm{~kg} / \mathrm{ha})$. En 2015 , ce sont les génotypes ISV128 $(178,68 \mathrm{~kg} / \mathrm{ha})$, Suvita2 (172,79 kg/ha) et IT96D-610 (73,61 kg/ha) qui ont donné les meilleurs rendements en graines.

Le poids de cent graines était légèrement plus élevé en 2015qui correspond à l'année la plus productive par rapport à 2014. Il a été très significativement affecté par les génotypes en 2014, et. Tiligré, IT93K-5031, et IT90K-284-2 ont les valeurs moyennes de PCG les plus élevés pour les deux années. Au niveau de la densité de densité, les PCG sont également inférieurs en 2015, avec des valeurs moyennes les plus élevées à la densité1.Tout comme le poids de cent graines, l'indice de récolte présente des valeurs moyennes les plus faibles en 2014 (3,74\%) par rapport à 2015 $(5,62 \%)$.Une différence très significative entre les génotypes a été enregistrée au cours des deux années. Par contre, la différence entre les densités de semis n'était pas significative en ce qui concerne l'indice de 
récolte. L'interaction génotype $\mathrm{x}$ densité pour l'indice de récolte n'a été significative qu'en 2015.

Tableau2 : Effet des génotypes et de la densité de semis de niébé sur les rendements grains et biomasse du mil en 2014 et2015

\begin{tabular}{|c|c|c|c|c|}
\hline \multirow[b]{2}{*}{ Génotypes } & \multicolumn{2}{|c|}{ Biomasse kg/ha } & \multicolumn{2}{|c|}{ Grains kg/ha } \\
\hline & 2014 & 2015 & 2014 & 2015 \\
\hline ISV128 & 1090,00 & 1338,65 & 107,95 & 195,72 \\
\hline ISV20 & 1416,11 & 1432,03 & 124,05 & 332,45 \\
\hline IT82E18 & 1222,92 & 1328,13 & 179,21 & 316,25 \\
\hline IT90K-284-2 & 1111,50 & 1455,56 & 171,79 & 291,11 \\
\hline IT93K-503-1 & 1144,96 & 1198,33 & 192,08 & 204,99 \\
\hline IT96D-610 & 1167,22 & 1200,55 & 106,72 & 371,53 \\
\hline IT98K-428-3 & 1090,23 & 1140,20 & 127,90 & 239,38 \\
\hline Mouride & 1105,22 & 1486,21 & 179,91 & 224,63 \\
\hline Suvita2 & 1059,72 & 1365,98 & 181,14 & 283,92 \\
\hline Tiligré & 1276,81 & 1556,75 & 196,56 & 289,80 \\
\hline Moyennes & 1168,47 & 1350,24 & 156,73 & 274,98 \\
\hline Témoin & 1899,67 & 1590,42 & 244,33 & 270,00 \\
\hline \multicolumn{5}{|l|}{ Densité } \\
\hline D1 & $1366,44^{\mathrm{a}}$ & 1365,66 & 177,99 & 299,94 \\
\hline D2 & $1033,83^{\mathrm{b}}$ & 1353,93 & 138,90 & 249,25 \\
\hline Génotypes & ns & ns & ns & ns \\
\hline Densité & $*$ & ns & ns & ns \\
\hline Génotype×Densité & $* *$ & $* * * *$ & ns & ns \\
\hline
\end{tabular}

$*, * *, * * *=$ significatifs au seuil de probabilité respectivement de $0,05,0,01$ et 0,$00 ; \mathrm{ns}=$ non significatif ( $p>0,05)$. Les chiffres portant la (ou les) même(s) lettre(s) dans la même colonne ne sont pas significativement différents au seuil de $\mathrm{p}<0,05$. 
Tableau3 :Effet des génotypes et de la densité de semis de niébé sur les rendements graines et biomasse du niébé en 2014 et2015

\begin{tabular}{|c|c|c|c|c|c|c|c|c|}
\hline \multirow[b]{2}{*}{ Génotypes } & \multicolumn{2}{|c|}{ Biomasse (kg/ha) } & \multicolumn{2}{|c|}{ Graines (kg/ha) } & \multicolumn{2}{|c|}{ PCG (g) } & \multicolumn{2}{|c|}{ IR (\%) } \\
\hline & 2014 & 2015 & 2014 & 2015 & 2014 & 2015 & 2014 & 2015 \\
\hline & $1047,19^{\mathrm{a}}$ & & & 178,68 & 12,50 & & $4,45^{\mathrm{abc}}$ & \\
\hline ISV128 & b & $1211,08^{\mathrm{ab}}$ & $83,68^{\mathrm{a}}$ & a & $\mathrm{c}$ & $12,79^{\mathrm{c}}$ & d & $7,35^{\mathrm{bc}}$ \\
\hline ISV20 & $771,94^{\mathrm{abc}}$ & $1226,08^{\mathrm{ab}}$ & $14,8^{\mathrm{bc}}$ & $46,49^{\mathrm{b}}$ & $\begin{array}{c}13,44 \\
\mathrm{c} \\
11,33\end{array}$ & $14,80^{\mathrm{bc}}$ & $0,62^{\mathrm{d}}$ & $1,93^{\mathrm{c}}$ \\
\hline IT82E18 & $517,92^{\mathrm{c}}$ & $698,52^{\mathrm{bcd}}$ & $\begin{array}{c}2,38^{\mathrm{c}} \\
40,29^{\mathrm{ab}}\end{array}$ & $21,14^{\mathrm{b}}$ & $\begin{array}{c}c \\
16,05\end{array}$ & $20,25^{\mathrm{a}}$ & $0,20^{\mathrm{d}}$ & $3,46^{\mathrm{c}}$ \\
\hline IT90K-284-2 & $798,33^{\mathrm{abc}}$ & $492,01^{\mathrm{cd}}$ & $\mathrm{c}$ & $18,67^{\mathrm{b}}$ & $\begin{array}{c}\text { b } \\
16,75\end{array}$ & $22,42^{\mathrm{a}}$ & $8,38^{\text {ad }}$ & $2,77^{\mathrm{c}}$ \\
\hline IT93K-503-1 & $586,01^{\mathrm{c}}$ & $1374,27^{\mathrm{a}}$ & $\begin{array}{l}64,53^{\mathrm{c}} \\
18,31^{\mathrm{ab}}\end{array}$ & $35,32^{\mathrm{b}}$ & b & $\begin{array}{c}19,5^{\mathrm{a}} \\
17,08^{\mathrm{ab}}\end{array}$ & $1,35^{\mathrm{d}}$ & $2,08^{c}$ \\
\hline IT96D-610 & $462,53^{\mathrm{c}}$ & $545,92^{\mathrm{cd}}$ & c & $73,61^{\mathrm{b}}$ & $16,4^{\mathrm{b}}$ & $\begin{array}{c}c \\
14,77^{\mathrm{ab}}\end{array}$ & $7,80^{\mathrm{abc}}$ & $13,93^{\mathrm{a}}$ \\
\hline IT98K-428-3 & $783,92^{\mathrm{abc}}$ & $443,96^{\mathrm{cd}}$ & $14,82^{\mathrm{bc}}$ & $16,34^{\mathrm{b}}$ & - & $\mathrm{c}$ & $1,25^{\mathrm{d}}$ & $8,33^{\mathrm{abc}}$ \\
\hline Mouride & $648,09^{\mathrm{bc}}$ & $376,46^{\mathrm{d}}$ & $17,88^{\mathrm{bc}}$ & $\begin{array}{l}20,28^{\mathrm{b}} \\
172,79\end{array}$ & c & $\begin{array}{l}18,79^{\mathrm{ab}} \\
17,32^{\mathrm{ab}}\end{array}$ & $1,62^{\mathrm{cd}}$ & $\begin{array}{l}2,70^{\mathrm{bc}} \\
10,67^{\mathrm{a}}\end{array}$ \\
\hline Suvita2 & $525,35^{\mathrm{c}}$ & $\begin{array}{l}841,42^{\text {abcd }} \\
1089,38^{\text {ab }}\end{array}$ & $92,69^{\mathrm{a}}$ & a & $16,6^{\mathrm{b}}$ & c & $8,88^{\mathrm{a}}$ & b \\
\hline Tiligré & $1076,74^{\mathrm{a}}$ & $\mathrm{c}$ & $66,85^{\mathrm{ab}}$ & $39,47^{\mathrm{b}}$ & $20,9^{\mathrm{a}}$ & $21,98^{\mathrm{a}}$ & $2,83^{\mathrm{bcd}}$ & $2,96^{\mathrm{c}}$ \\
\hline Moyennes & 721,80 & 829,91 & 41,62 & 62,28 & 15,20 & 18,00 & 3,74 & 5,62 \\
\hline Densité & & & & & & & & \\
\hline D1 & $609,58^{\mathrm{b}}$ & $660,56^{\mathrm{b}}$ & $36,22^{\mathrm{a}}$ & $68,70^{\mathrm{a}}$ & $\begin{array}{c}16,92 \\
\text { a } \\
15,74\end{array}$ & $17,85^{\mathrm{a}}$ & $3,76^{\mathrm{a}}$ & $6,69^{\mathrm{a}}$ \\
\hline D2 & $834,03^{\mathrm{a}}$ & $1137,47^{\mathrm{a}}$ & $52,92^{\mathrm{a}}$ & $83,64^{\mathrm{a}}$ & $\mathrm{a}$ & $16,09^{\mathrm{a}}$ & $3,65^{\mathrm{a}}$ & $5,14^{\mathrm{a}}$ \\
\hline Génotypes & $*$ & $*$ & $* *$ & $* * * *$ & $* *$ & $* * *$ & $*$ & $* * *$ \\
\hline $\begin{array}{c}\text { Densité } \\
\text { Génotype } \times \text { Densit }\end{array}$ & * & $* *$ & ns & ns & ns & ns & ns & ns \\
\hline é & $* *$ & * & $* *$ & $* * *$ & * & ns & ns & $* *$ \\
\hline
\end{tabular}

\section{Discussion}

L'examen des résultats de la culture associée mil-niébé, a permis de mettre en évidence les effets du génotype et de la densité sur le rendement en graines, biomasses et poids cent graines chez le niébé. L'effet génotype a significativement affecté tous les paramètres mesurés chez le niébé .Les rendements en graines ont relativement augmenté pour tous les génotypes en 2015. Les faibles rendements en 2014 pour le niébé, peuvent s'expliquer par le semi tardif. L'augmentation de la densité de semis a augmenté les rendements en graines et biomasse du niébé. Ce résultat s'explique par le fait que lorsque la densité des plants par $\mathrm{m}^{2}$ augmente les rendements par plants 
diminuent, tandis que ceux par unité de surface augmentent (Ahmed et al., 2012 ; Khamooshi et al., 2012 ; Derogar et Mojjadam, 2014).

Le poids de cent graines a été significativement affecté par les génotypes. La faible densité de semis (densité 1), a entrainé une augmentation du poids de cent graines. Ces résultats sont conformes à ceux trouvés par Ahmed et Abdelrhim (2010) ; Ahmed et al., (2012), qui ont montré que le poids de cent graines était plus élevé pour les faibles densités. Cela peut être lié à la faible compétition entre les plantes pour les éléments nutritifs, l'eau ainsi qu'une meilleure efficacité de la photosynthèse. L'indice de récolte en grains du niébé a aussi été très significativement $(\mathrm{p}<0,001)$ affecté par les génotypes de niébé. Il est généralement plus élevé en densité 1 et plus faible en densité 2 à cause de la production de biomasse élevée à cette densité. Des résultats similaires ont été obtenus par Weber et al. (1966) qui ont rapporté que les faibles densités de semis ont tendance à augmenter l'indice de récolte.

Parmi les génotypes de niébé étudiés, ISV128 a donné le rendement moyen en graines le plus élevé. Ce génotype fait aussi partie de ceux qui ont le meilleur rendement en biomasse. Il peut de ce fait être considéré comme étant à double fins:pour la production des graines destinées à la consommation et pour l'intégration agriculture-élevage en zone sahélienne, car les fanes de niébé constituent une importante source de protéines pour le bétail.

L'effet génotype n'a eu aucune influence sur la productivité du mil, ce qui corrobore les résultats de Mohammed et al.(2008) qui n’ont trouvé aussi aucun effet génotype sur la biomasse et le rendement en grains du sorgho en association avec le niébé. Mbaye et al.(2014), travaillant sur différentes dates et densités de semis du niébé associé au mil n'ont trouvé aucun effet significatif du niébé sur la productivité du mil et ont montré que les résultats différent d'une année à l'autre en fonction des dates et des densités de semis.

Les résultats indiquent que les rendements sont plus élevés en 2015 qu'en 2014 à cause du semis tardif réalisé en première année. Les génotypes n'ont pas donc bénéficié des conditions optimales de pluviométrie. En 2014, l'association du mil avec le niébé s'est traduite par un rendement plus faible en biomasse et en grain à la densité 2. En effet, les deux cultures sont en compétition pour les ressources du milieu notamment l'eau, les éléments minéraux et la lumière. Selon Dalal (1977), Wahua et Miller (1978), Wanki et al. (1982), la croissance de deux ou plusieurs cultures en association se traduit souvent par une diminution des rendements de deux cultures en raison de la concurrence pour les ressources essentielles limitées. Ainsi, la meilleure façon de réduire la concurrence entre deux cultures en association pour les éléments minéraux et l'eau, c'est de décaler le semis entre les deux 
cultures dans un système en intercalaire (Andrews et Kassam, 1976). Cependant, ce système favoriserait plus la céréale (Ntare, 1989, 1990 ;Reddy et visser, 1997 ; Olufajo et al. 2008 ; Hide Omae et al. 2014) à cause de sa plus forte compétitivité pour l'utilisation de l'azote du sol et de la lumière. Elle peut donc réduire la croissance du niébé qui se traduit par la réduction $\mathrm{du}$ rendement en matière sèche en raison de l'ombrage. La meilleure stratégie de réduire la dominance du mil et optimiser la productivité du niébé c'est de semer simultanément les deux cultures (Singh et Ajeigbe, 2000).

L'effet positif de l'association céréales/légumineuses à graines sur la céréale est rarement obtenu à la prochaine année (Garba et Renard, 1991). La performance des associations en 2015 dans le cadre de cette étude peut s'expliquer par l'effet résiduel dû à la fixation symbiotique de l'azote atmosphérique par les légumineuses. L'azote atmosphérique fixé par le niébé en première année n'a donc été bénéfique que pour la deuxième année surtout pour les grains.

\section{Conclusion}

L'évaluation des rendements chez le niébé suivant deux densités en association avec le mil a donné des résultats plus intéressants avec la densité 2. Cette densité a augmenté les rendements du niébé sans affecter significativement la productivité du mil pendant les deux années. Par ailleurs, l'augmentation du nombre de plants de niébé $/ \mathrm{m}^{2}$ peut contribuer à obtenir un effet positif de l'association dès la deuxième année de culture.

Cependant, on note une différence inter-génotypes très significative pour tous les paramètres mesurés chez le niébé.

$\mathrm{Au}$ terme de cette étude, nous déduisons que dans le cadre de 1'association avec le mil, ISV128 et Suvita2 seraient aptes pour la production en graines, ISV128, Tiligré, et ISV20 pour la production en biomasse. IT98K-428-3 est le génotype le moins performant et ne présentant aucun intérêt pour l'association mil/niébé.

\section{References:}

Ahmed M.E.N and Abdelrhim A.J. (2010). Effect of Plant density and Cultivar on Growth and Yield of Cowpea (Vigna unguiculata L.Walp) Australian Journal of Basic and Applied Sciences, 4(8): 3148-3153

Ahmed M.E.N, Abdelrhim A.J, Salaheldeen E.A, Feisal M.I, Elshiekh A.I. (2012).Determination of Suitable Variety and Plants per Stand of Cowpea (Vigna Unguiculata L.Walp) in the Sandy Soil, Sudan. Advances in Life Sciences, 2(1): 1-5

Andrew D.J. and A.H. Kassam. (1976). The importance of multiple cropping increasing world food supplies, (p. 1-11).In: R.I. Papendick, P.A. Sanchez, 
and G.B.Triplett (eds.). Multiple cropping. Amer.Soc. Agron., Madison, Wis. Spec. Publ.27.

Atta S., Moutari M.A., Moutari A., François A. and Mahamane S. (2011).Interannual variation in fodder production in cowpea varieties in Niger. International Journal of Biological and Chemical Sciences, 5(4):16591675

Bationo A, Lompo F, and Kaola S. (1998). Research on nutrient flours and balances invest state of the art. Agric. Ecosyst. Environ. 71: 19-36.

Bationo. A and Ntare. B. R. (2000). Rotation and nitrogen fertilizer effects on pearl millet, cowpea and groundnut yield and soil chemical properties in a sandy soil in the semi-arid tropics, West Africa. Journal of Agricultural science, Cambridge. 134, 277-284.

Boubié V.B. (2002). Rôle des légumineuses sur la fertilité des sols ferrugineuxtropicaux des zones guinéenne et soudanienne du Burkina Faso. Thèse de doctorat del'Université Laval Québec. 197p.

Caroline D.D. (2006). Impact de pratiques de gestion de fertilité sur lesrendements en mil dans le fakara (Niger). Travail de fin d'études présenté en vue del'obtention du grade de bio-ingénieur. Université de Louvain. 214p. Dalal R.C. (1977).Effect of intercropping on maize with soya bean on grain yield. Trop. Agr. (Trinidad) 54:189-191.

Derogar N and Mojaddam M. (2014). Effect of plant density on grain yield and yield components in faba bean. International journal of plant, animal and environnemental science

(IJPAES). Volume-4, Issue-2, April-June. p. 93-96.

Elodie B. (2012). Interaction entre céréales et légumineuses en association et acquisition de phosphore du sol : processus rhizosphériques sous-jacents. Thèse de doctorat. Centre international d'études supérieures en sciences agronomiques (Montpellier Sup Agro).P. 244.

GARBA M and RENARD C. (1991). Biomass production. Yields and water use efficiency in some pearl millet legumes cropping system at Sadoré (Niger) in Proceedings International workshop on soil water balance in the sudano-sahelian zone, 18-23 February 1991 (ed. Sivakumar M.V.K., Wallace J.S., Rebard C. and Giroux C.), Niamey), pp 431-439.

Hide O, Adam K.S and Satoshi T. (2014). Improving Millet-Cowpea Productivity and soil Fertility with Crop Rotation, Row Arrangement and Cowpea Density in the Sahel, West Africa. American-Eurasian J. Agric \&Environ. Sci., 14 (2): 110-115, 2014.

JAICAF. 2009. Les céréales au Niger. Accent sur le mil et le sorgho. Association pour la Collaboration Internationale en Matière d'Agriculture et de Forêts du Japon. Tokyo, Japon, 109p. 
Jérôme P, Pierre D, Christian R, Bernard B. (2013). Perspectives offertes par la culture en association de froment et de pois protéagineux d'hiver.13 $3^{\text {ème }}$ Journée productions porcines et avicoles. P. 25-32.

Khamooshi H, Mohammadian N, Saamdaliri M, ForoughiZ. (2012).Study of effect plant density and nitrogen on yield components of Visia faba (Faba Bean). Journal of Ornamental and Horticultural Plants, 2 (3): p. 161-167.

Lina S, Irena D, Ausra A, Zydré K, Stanislava M. (2012). Pea and spring cereal intercropping Systems: Advantages and suppression of Broad-Leaved Weeds. Pol. J. Environ. Stud. Vol. 22, No 2 (2013), p. 541-551.

Manfred H. (1993). Harvest index versus grains/straw-ratio. Theoretical comments and experimental results on the comparison of variation. Euphytica 68: 27-32, 1993.

Mbaye M.S, Kane A, Gueye M, Bassene C, Diop D.B.N, Sylla S.N, Noba K. (2014).

Date et densité optimales de semis du niébé [Vigna unguiculata(L.) Walp.] en associationavec le mil [Pennisetum glaucum (L.) R. Br.]. Journal of Applied Biosciences 76: p. 6305-6315.

Mohammed I.B, Olufajo O.O, Singh B.B, Miko S, Mohammed S.G. (2008). Evaluation of yield of components of sorghum/cowpea intercrops in the Sudan savanna ecological zone. ARPN J. Agric. Biol.Sci., 3(3): 30-37.

Naudin C, Corre-Hellou G, Protin P.-V, Trochard R. (2009). Impact des pratiques de fertilisation sur la productivité des prairies et mélanges céréalesprotéagineux et la qualité du fourrage. Fourrages.198, p.115-130.

Ntare, B.R.(1989). Evaluation of Cowpea Cultivars for Intercropping with Pearl Millet in theSahelian Zone of West Africa. Field Crops Res., 20: 31-40. Ntare, B.R.(1990).Intercropping morphologically different cowpeas with pearl millet in ashort season environment in the Sahel. Expl Agric. (1990), volume 26, pp.41-47.

Olufajo O.O, I.B. Mohammed, Singh B.B, Oluwasemire K.O. and Chiezey U.F. (2008). Productivity of millet/ cowpea intercrops as affected by cowpea genotype and rowarrangement. World of Journal Agricultural Sciences 4 (S): p.818-824.

Reddy. K.C and Visser P.L. (1997). Cowpea Intercrop Growth and yield as affected by timeof planting relative to millet. African Crop Sci. J., 5: 351357.

Singh B.B. and Ajeigbe H.A. (2000). Improving cowpea-cereals based cropping. Pages 278-286 in Challenges and Opportunies for enhancing sustainable Cowpea Production, edited by C.A. Fatokun, S.A. Tarawali, B.B. Singh, P.M. Kormawa, and Tamo. Systems in the dry savanna of West Africa. Challenges and opportunities for enhancing sustainable cowpea production. Proceedings of the World cowpea conference III held at the 
International Instituteof Tropical Agriculture (IITA), Ibadan, Nigeria 4-8 September 2000. IITA, Ibadan, Nigeria.

$396 \mathrm{p}$.

Yamoah C.F, Bationo A, Shapiro B, and Koala S. (2003). Soil management practices to

Improve nutrient use efficiencies and reduce risk in millet-based cropping systems in the

Sahel. Tropicultura, 21, 2, p. 66-72.

Weber C.R, Shibles R.M and Byth D.E. (1966). Effect of plant population and row spacing on Soybean development and production. American Society of Agronomy Journal, Vol. (58):99-102, Madison, U.S.A

Wahua T.A.T. and Miller D.A. (1978). Relative yield totals and yield components of intercropped sorghum and soybeans. Agron. J. 70:287-291.

Wanki S.B.C, M.O.A. Fawusi, and D. Nangju. (1982). Pod and grain yields from intercropping maize and Vigna unguiculata (L) Walp in Nigeria. J. Agr. Sci. 99:13-17. 